\title{
BAERLIN2014 - stationary measurements and source apportionment at an urban background station in Berlin, Germany
}

Erika von Schneidemesser et al.

Correspondence to: Erika von Schneidemesser (evs@iass-potsdam.de)

The copyright of individual parts of the supplement might differ from the CC BY 4.0 License. 


\section{Section S1. NMVOC data:}

As stated in the main text of the paper, to be able to make reasonable comparisons with previous work regarding the contribution of different compound classes to the measured mixing ratios of NMVOCs, as well as the $\mathrm{OH}$ reactivity attributed to these NMVOCs, a subset of the compounds was selected and used in the analysis. This subset was based on a number of different papers in the literature, and those compounds that were regularly included in OH reactivity calculations (e.g., (Dolgorouky et al., 2012; Gilman et al., 2009; Goldan et al., 2004; Liu et al., 2008)). This includes 57 NMVOCs, and while this does not capture the complete $\mathrm{OH}$ reactivity, it allows for more equal comparisons with other studies. The 57 NMVOCs included, are as follows:

Alkanes: cyclohexane, cyclopentane/2,3-dimethylbutane, ethane, heptane, methylcyclohexane, methylcyclopentane, n-decane, n-hexane, n-octane, n-pentane, propane, 2-methylbutane, 2-methylheptane, 2methylhexane, 2-methylpentane, 2-methylpropane, 2,2-dimethylbutane, 2,3-dimethylpentane, 3-methylpentane

Alkenes and alkynes: cis-2-butene, cis-2-pentene, ethyne, i-butene, 1-butene, propene, trans-2-butene, trans-2pentene, 1-pentene, 1,3-butadiene, 2-methyl-1-butene, 2-methyl-2-butene, 3-methyl-1-butene

Aromatics: benzene, ethylbenzene, i-propylbenzene, m-ethyltoluene, m-/p-xylene, n-propylbenzene, oethyltoluene, o-xylene, p-ethyltoluene, styrene, toluene, 1,2,3-trimethylbenzene, 1,2,4-trimethylbenzene/tbutylbenzene, 1,3,5-trimethylbenzene

Oxygenated: acetaldehyde, acetone, ethanol, MEK, methanol, 2-propanol

Biogenics and their oxidation products: $\alpha$-pinene, $\beta$-pinene, isoprene, methacrolein, methylvinylketon

For information on individual mixing ratios by location, see Bonn et al. 2016. 
Table S1. Details of the NMVOC measurements.

\begin{tabular}{|c|c|c|c|}
\hline Instrument & Compounds or $\mathrm{m} / \mathrm{z}$ measured & Detection limits & $\begin{array}{l}\text { Sampling } \\
\text { interval }\end{array}$ \\
\hline PTR-MS & $\begin{array}{l}28,29,31,33,34,35,38,39,41,42,43,44,45,46,47,48,49,50,51,53,55,56,57,59,60,61,63,65,67,69, \\
71,73,75,77,79,81,83,85,86,87,89,91,93,95,97,99,101,103,105,107,109,113,115,117,119,121, \\
127,129,132,135,137,143,147,149,151,155,157,181,205\end{array}$ & $\begin{array}{l}\text { varies (10s of pptv), } \\
\text { see Bourtsoukidis et } \\
\text { al., } 2012\end{array}$ & 4.5 minutes \\
\hline $\begin{array}{l}\text { AMA Instruments } \\
\text { GC5000 BTX }\end{array}$ & benzene, toluene & 30 pptv & 30 min data \\
\hline Canister samples & $\begin{array}{l}\text { Ethene, Ethyne, Ethane, Propene, Propane, Propyne, Acetaldehyde, 2-Methylpropane, Methanol, 1-Butene/i- } \\
\text { butene, 1,3-Butadiene, n-Butane, trans-2-Butene, cis-2-Butene, 1,2-Butadiene, Ethanol, 3-Methyl-1-butene, 2- } \\
\text { Methylbutane, Acetone, 1-Pentene, 2-Propanol, 2-Methyl-1-butene, n-Pentane, Isoprene, trans-2-Pentene, cis-2- } \\
\text { Pentene, Propanal, 2-Methyl-2-butene, Acetic acid methyl ester, 1,3-Pentadiene, Cyclopentadiene, 2,2- } \\
\text { Dimethylbutane, 2-Butanol, 1-Propanol, Cyclopentene, Methacrolein, Cyclopentane/2,3-dimethylbutane, 2- } \\
\text { Methylpentane, Methyl vinyl ketone, Butanal, 1-Hexene, 3-Methylpentane, 2-Methyl-1-pentene, n-Hexane, } \\
\text { trans-2-Hexene, cis-2-Hexene, 1,3-Hexadiene, Methylcyclopentane, 2,4-Dimethylpentane, Methylcyclopentene, } \\
\text { Benzene, 1-Butanol, Cyclohexane, 2-Methylhexane, 2,3-Dimethylpentane, 3-Methylhexane, Pentanal, } \\
\text { Cyclohexene, 1,3-Dimethylcyclopentane, 1-Heptene, 2,2,4-Trimethylpentane, Heptane, 2,3-Dimethyl-2-pentene, } \\
\text { Octene, Methylcyclohexane, 2,3,4-Trimethylpentane, Toluene, 2-Methylheptane, 4-Methylheptane, 3- } \\
\text { Methylheptane, Hexanal, Acetic acid butyl ester, n-Octane, Dimethylcyclohexane isomer, Ethylbenzene, m/p- } \\
\text { Xylene, Heptanal, Styrene, 1-Nonene, o-Xylene, n-Nonane, i-Propylbenzene, } \alpha \text {-Pinene, n-Propylbenzene, m- } \\
\text { Ethyltoluene, p-Ethyltoluene, 1,3,5-Trimethylbenzene, Sabinene, o-Ethyltoluene, Octanal, } \\
\beta \text {-Pinene, 1,2,4-Trimethylbenzene/t-butylbenzene, n-Decane, 1,2,3-Trimethylbenzene, Limonene, Eucalyptol, } \\
\text { Indane, 1,3-Diethylbenzene, 1,4-Diethylbenzene, Butylbenzene, n-Undecane, n-Dodecane, n-Tridecane }\end{array}$ & $\begin{array}{l}\text { 5-20 ppt (see Urban } \\
\text { 2010, Table } 3.3 \text { for } \\
\text { individual LOD) }\end{array}$ & $\begin{array}{l}\text { Intermittent (non- } \\
\text { continuous) } \\
\text { samples }\end{array}$ \\
\hline Cartridge samples & $\begin{array}{l}\text { Isoprene, 2-methyl-3-butenol, } \alpha \text {-pinene, camphene, } \beta \text {-pinene, } 3 \Delta \text {-carene, p-cymene, 1,8-cineol, limonene, } \\
\text { terpinolene, 4-acetyl-1-methylcyclohexene, nopinone, bornylacetate, longicyclene, iso-longifolene, } \beta \text { - } \\
\text { caryophyllene, aromadendrene, } \alpha \text {-humulene, Benzene, Toluene, Ethylbenzene, } \mathrm{p} / \mathrm{m} \text {-xylene, styrene, o-xylene, } \\
\text { propylbenzene, 3-ethyltoluene, 4-ethyltoluene, 1,3,5-trimethylbenzene, } 2 \text {-ethyltoluene, 1,2,4-trimethylbenzene, } \\
\text { 1,2,3-trimethylbenzene, hexane, heptane, octane, nonane, decane }\end{array}$ & $\begin{array}{l}\text { 3-60 } \mathrm{ng} / \mathrm{m}^{3} \text { (see } \\
\text { main text and Mäki } \\
\text { et al. (2017) for } \\
\text { more details) }\end{array}$ & $\begin{array}{l}\text { Intermittent (non- } \\
\text { continuous) } \\
\text { samples }\end{array}$ \\
\hline
\end{tabular}


Table S2. Pearson correlation coefficients (r) with 95\% confidence intervals for hourly data.

\begin{tabular}{|c|c|c|c|c|c|c|c|c|c|c|c|}
\hline & $\begin{array}{l}\text { RH } \\
(\%)\end{array}$ & $\begin{array}{c}\mathrm{O}_{3} \\
\left(\mu \mathrm{g} \mathrm{m}^{-3}\right)\end{array}$ & $\begin{array}{c}\mathrm{NO} \\
\left(\mu \mathrm{g} \mathrm{m}^{-3}\right)\end{array}$ & $\begin{array}{c}\mathrm{NO}_{2} \\
\left(\mu \mathrm{g} \mathrm{m}^{-3}\right)\end{array}$ & $\begin{array}{c}\mathrm{CO} \\
\left(\mathrm{mg} \mathrm{m}^{-3}\right)\end{array}$ & $\begin{array}{l}\text { Benzene } \\
\left(\mu \mathrm{g} \mathrm{m}^{-3}\right)\end{array}$ & $\begin{array}{l}\text { Toluene } \\
\left(\mu \mathrm{g} \mathrm{m}^{-3}\right)\end{array}$ & $\begin{array}{c}\mathrm{PM}_{10} \\
\left(\mu \mathrm{g} \mathrm{m}^{-3}\right)\end{array}$ & $\begin{array}{c}\text { MLH } \\
(\mathrm{m})\end{array}$ & $\begin{array}{l}\text { PKW at } \\
\text { MC220 } \\
\text { (count) } \\
\end{array}$ & $\begin{array}{l}\text { PKW at } \\
\text { MC143 } \\
\text { (count) } \\
\end{array}$ \\
\hline $\begin{array}{l}\text { Temp } \\
\left({ }^{\circ} \mathrm{C}\right)\end{array}$ & $\begin{array}{c}-0.71 \\
{[-0.72,-} \\
0.70]\end{array}$ & $\begin{array}{c}0.82 \\
{[0.81,0.83]}\end{array}$ & $\begin{array}{c}-0.28 \\
{[-0.31,-} \\
0.26]\end{array}$ & $\begin{array}{c}-0.38 \\
{[-0.40,-} \\
0.35]\end{array}$ & $\begin{array}{c}-0.26 \\
{[-0.28,-} \\
0.23]\end{array}$ & $\begin{array}{c}-0.14 \\
{[-0.17,-} \\
0.11]\end{array}$ & $\begin{array}{c}-0.15 \\
{[-0.18,-} \\
0.13]\end{array}$ & $\begin{array}{c}0.36 \\
{[0.34,0.38]}\end{array}$ & $\begin{array}{c}0.50 \\
{[0.47,0.53]}\end{array}$ & $\begin{array}{c}0.60 \\
{[0.58,0.63]}\end{array}$ & $\begin{array}{c}0.55 \\
{[0.52,0.57]}\end{array}$ \\
\hline $\begin{array}{l}\mathrm{RH} \\
(\%)\end{array}$ & 1 & $\begin{array}{c}-0.76 \\
{[-0.77,-} \\
0.74]\end{array}$ & $\begin{array}{c}0.17 \\
{[0.15,0.20]}\end{array}$ & $\begin{array}{c}0.17 \\
{[0.14,0.20]}\end{array}$ & $\begin{array}{c}0.13 \\
{[0.10,0.15]}\end{array}$ & $\begin{array}{c}0.08 \\
{[0.06,0.11]}\end{array}$ & $\begin{array}{c}0.15 \\
{[0.13,0.18]}\end{array}$ & $\begin{array}{c}-0.18 \\
{[-0.21,-} \\
0.15]\end{array}$ & $\begin{array}{c}-0.66 \\
{[-0.69,-} \\
0.64]\end{array}$ & $\begin{array}{c}-0.74 \\
{[-0.75,-0.72]}\end{array}$ & $\begin{array}{c}-0.70 \\
{[-0.72,-} \\
0.68]\end{array}$ \\
\hline $\begin{array}{l}\mathrm{O}_{3} \\
\left(\mu \mathrm{g} \mathrm{m}^{-3}\right)\end{array}$ & & 1 & $\begin{array}{c}-0.39 \\
{[-0.41,-} \\
0.36]\end{array}$ & $\begin{array}{c}-0.57 \\
{[-0.58,-} \\
0.55]\end{array}$ & $\begin{array}{c}-0.43 \\
{[-0.45,-} \\
0.41]\end{array}$ & $\begin{array}{c}-0.33 \\
{[-0.35,-} \\
0.30]\end{array}$ & $\begin{array}{c}-0.34 \\
{[-0.36,-} \\
0.31]\end{array}$ & $\begin{array}{c}0.24 \\
{[0.22,0.27]}\end{array}$ & $\begin{array}{c}0.59 \\
{[0.57,0.62]}\end{array}$ & $\begin{array}{c}0.55 \\
{[0.52,0.58]}\end{array}$ & $\begin{array}{c}0.50 \\
{[0.47,0.53]}\end{array}$ \\
\hline $\begin{array}{l}\text { NO } \\
\left(\mu \mathrm{g} \mathrm{m}^{-3}\right)\end{array}$ & & & - & $\begin{array}{c}0.58 \\
{[0.56,0.59]}\end{array}$ & $\begin{array}{c}0.72 \\
{[0.70,0.73]}\end{array}$ & $\begin{array}{c}0.53 \\
{[0.51,0.55]}\end{array}$ & $\begin{array}{c}0.49 \\
{[0.47,0.51]}\end{array}$ & $\begin{array}{c}0.09 \\
{[0.06,0.11]}\end{array}$ & $\begin{array}{c}-0.20 \\
{[-0.24,-} \\
0.16]\end{array}$ & $\begin{array}{c}-0.06 \\
{[-0.11,-0.02]}\end{array}$ & $\begin{array}{c}0.00 \\
{[-0.04,0.04]}\end{array}$ \\
\hline $\begin{array}{l}\mathrm{NO}_{2} \\
\left(\mu \mathrm{g} \mathrm{m}^{-3}\right)\end{array}$ & & & & 1 & $\begin{array}{c}0.82 \\
{[0.81,0.83]}\end{array}$ & $\begin{array}{c}0.63 \\
{[0.62,0.65]}\end{array}$ & $\begin{array}{c}0.51 \\
{[0.49,0.53]}\end{array}$ & $\begin{array}{c}0.11 \\
{[0.08,0.14]}\end{array}$ & $\begin{array}{c}-0.29 \\
{[-0.33,-} \\
0.25]\end{array}$ & $\begin{array}{c}-0.06 \\
{[-0.10,-0.02]}\end{array}$ & $\begin{array}{c}-0.05 \\
{[-0.09,-} \\
0.01]\end{array}$ \\
\hline $\begin{array}{l}\mathrm{CO} \\
\left(\mathrm{mg} \mathrm{m}^{-3}\right)\end{array}$ & & & & & 1 & $\begin{array}{c}0.71 \\
{[0.70,0.72]}\end{array}$ & $\begin{array}{c}0.59 \\
{[0.57,0.60]}\end{array}$ & $\begin{array}{c}0.16 \\
{[0.14,0.19]}\end{array}$ & $\begin{array}{c}-0.22 \\
{[-0.26,-} \\
0.18]\end{array}$ & $\begin{array}{c}0.01 \\
{[-0.03,0.05]}\end{array}$ & $\begin{array}{c}0.02 \\
{[-0.02,0.06]}\end{array}$ \\
\hline $\begin{array}{l}\text { Benzene } \\
\left(\mu \mathrm{g} \mathrm{m}^{-3}\right)\end{array}$ & & & & & & 1 & $\begin{array}{c}0.79 \\
{[0.78,0.80]}\end{array}$ & $\begin{array}{c}0.23 \\
{[0.21,0.26]}\end{array}$ & $\begin{array}{c}-0.15 \\
{[-0.20,-} \\
0.11]\end{array}$ & $\begin{array}{c}0.02 \\
{[-0.02,0.06]}\end{array}$ & $\begin{array}{c}0.02 \\
{[-0.02,0.06]}\end{array}$ \\
\hline $\begin{array}{l}\text { Toluene } \\
\left(\mu \mathrm{g} \mathrm{m}^{-3}\right)\end{array}$ & & & & & & & 1 & $\begin{array}{c}0.17 \\
{[0.14,0.20]}\end{array}$ & $\begin{array}{c}-0.15 \\
{[-0.19,-} \\
0.10]\end{array}$ & $\begin{array}{c}-0.06 \\
{[-0.10,-0.01]}\end{array}$ & $\begin{array}{c}-0.07 \\
{[-0.11,-} \\
0.03]\end{array}$ \\
\hline $\begin{array}{l}\mathrm{PM}_{10} \\
\left(\mu \mathrm{g} \mathrm{m}^{-3}\right)\end{array}$ & & & & & & & & 1 & $\begin{array}{c}0.04 \\
{[-0.00,0.08]}\end{array}$ & $\begin{array}{c}0.19 \\
{[0.15,0.23]}\end{array}$ & $\begin{array}{c}0.12 \\
{[0.08,0.16]}\end{array}$ \\
\hline MLH (m) & & & & & & & & & 1 & $\begin{array}{c}0.53 \\
{[0.48,0.57]}\end{array}$ & $\begin{array}{c}0.48 \\
{[0.43,0.52]}\end{array}$ \\
\hline $\begin{array}{l}\text { PKW at } \\
\text { MC220 } \\
\text { (count) }\end{array}$ & & & & & & & & & & 1 & $\begin{array}{c}0.94 \\
{[0.93,0.94]}\end{array}$ \\
\hline
\end{tabular}



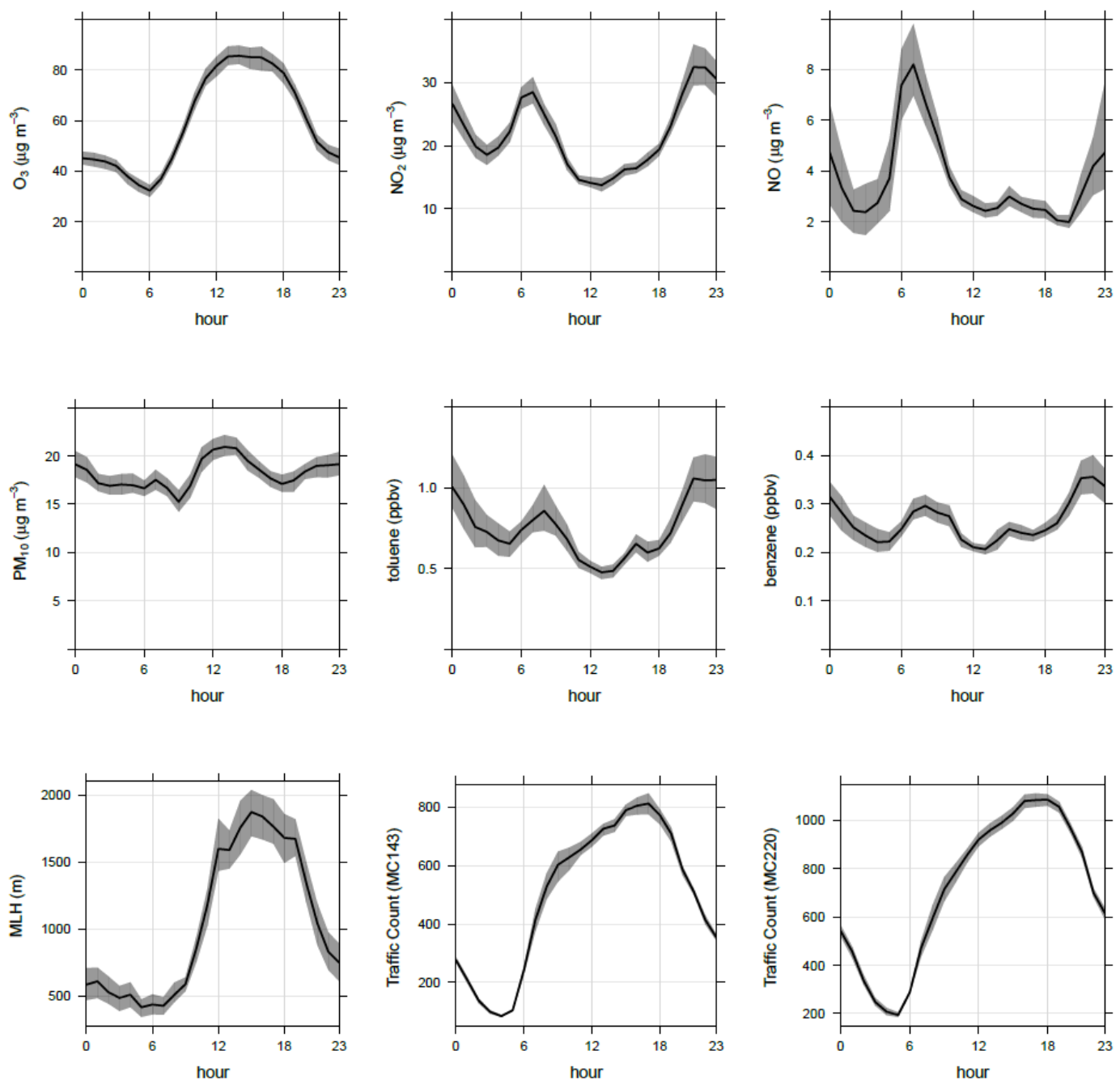

Figure S1. Diurnal variation in air pollutants and MLH measured in Neukölln, and traffic counts from the MC143 and MC220 locations. 


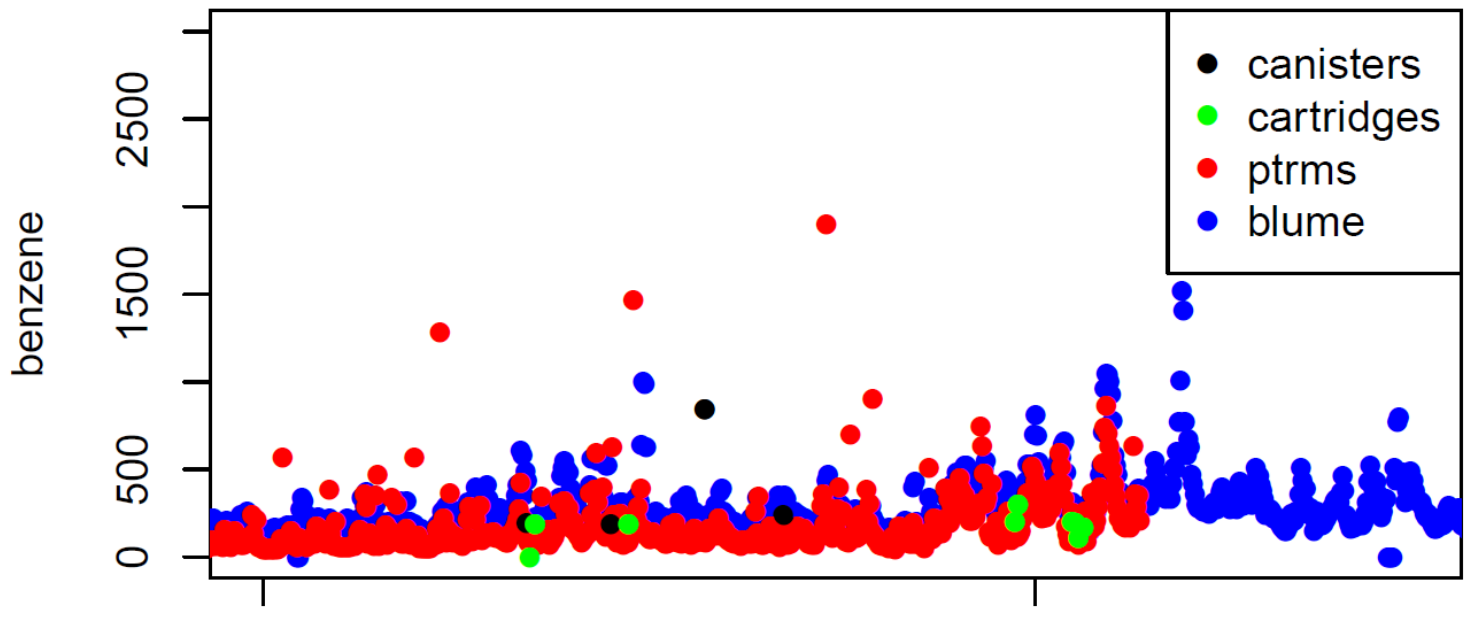

Aug 18

Aug 28

date

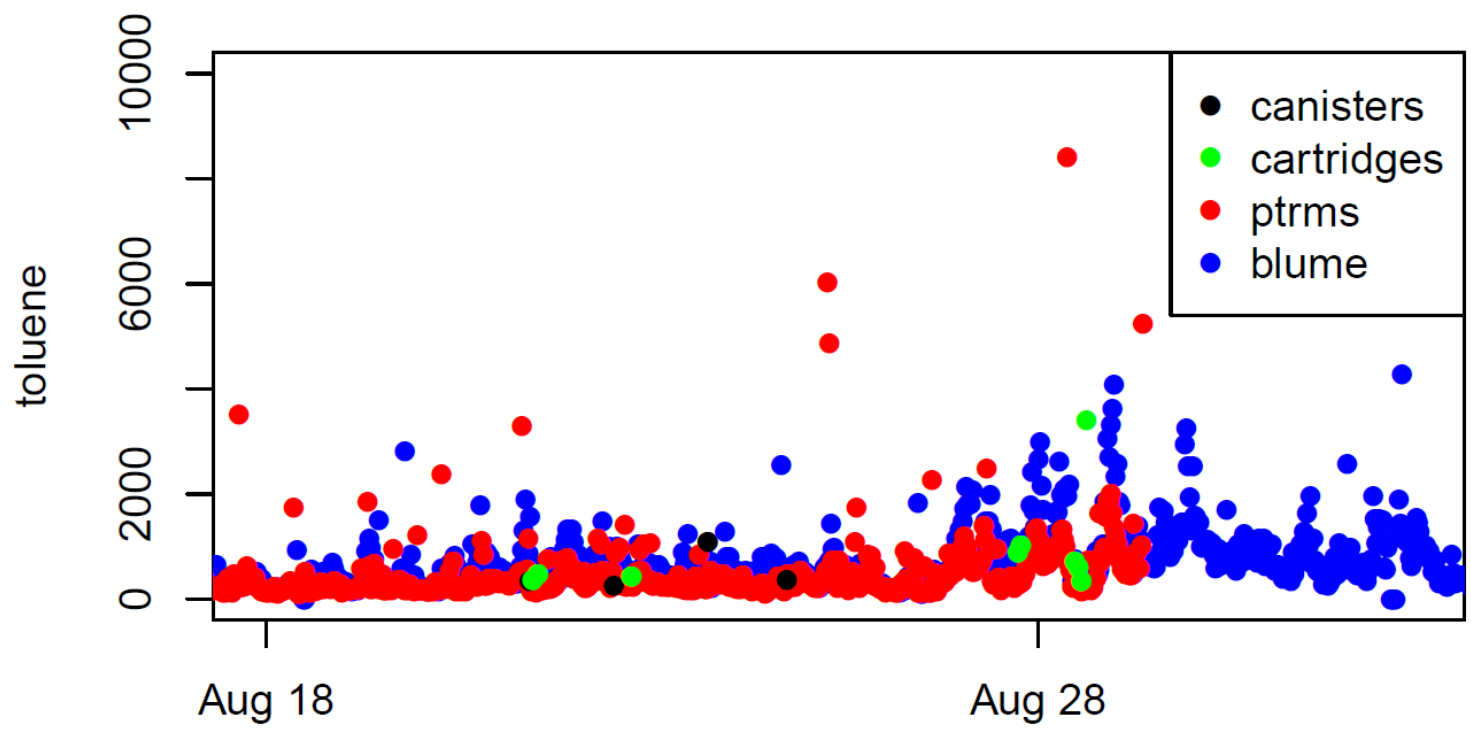

date

Figure S2. Mixing ratios measured for benzene (top) and toluene (bottom) by the individual cartridge and canister samples, and the continuous PTR-MS and BLUME network. Units are pptv. 

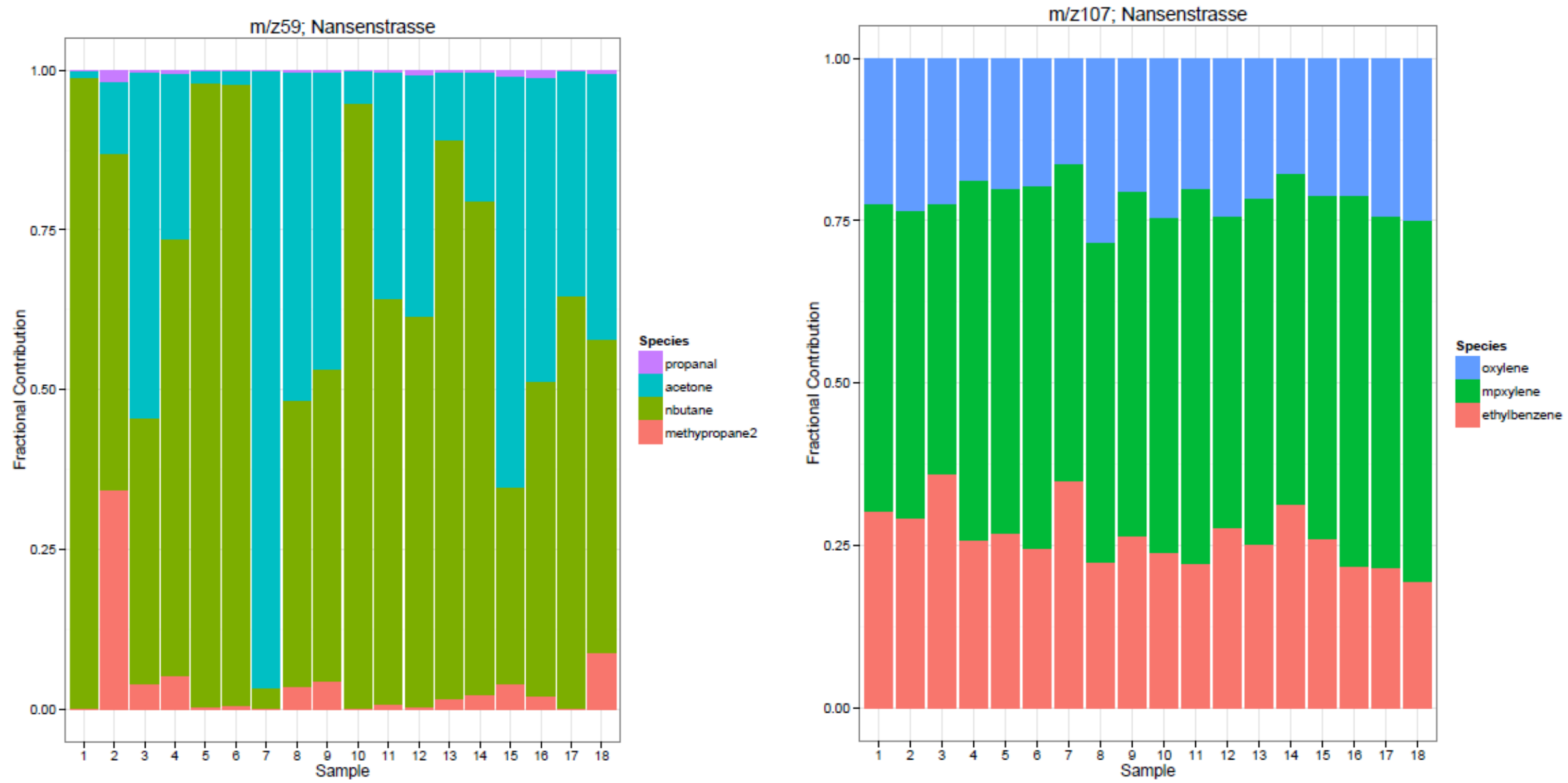

Figure S3. Fractional contribution of the individual NMVOCs measured by canister sample (GC-MS) to m/z 59 (left) and m/z 107 (right) for the 18 individual samples taken in Neukölln during the campaign. 
Figure S4. Back trajectories calculated by filter, organized by filter group.

(a) Group A

NOAA HYSPLIT MODEL

Backward trajectories ending at 0900 UTC 09 Jul 14

GDAS Meteorological Data

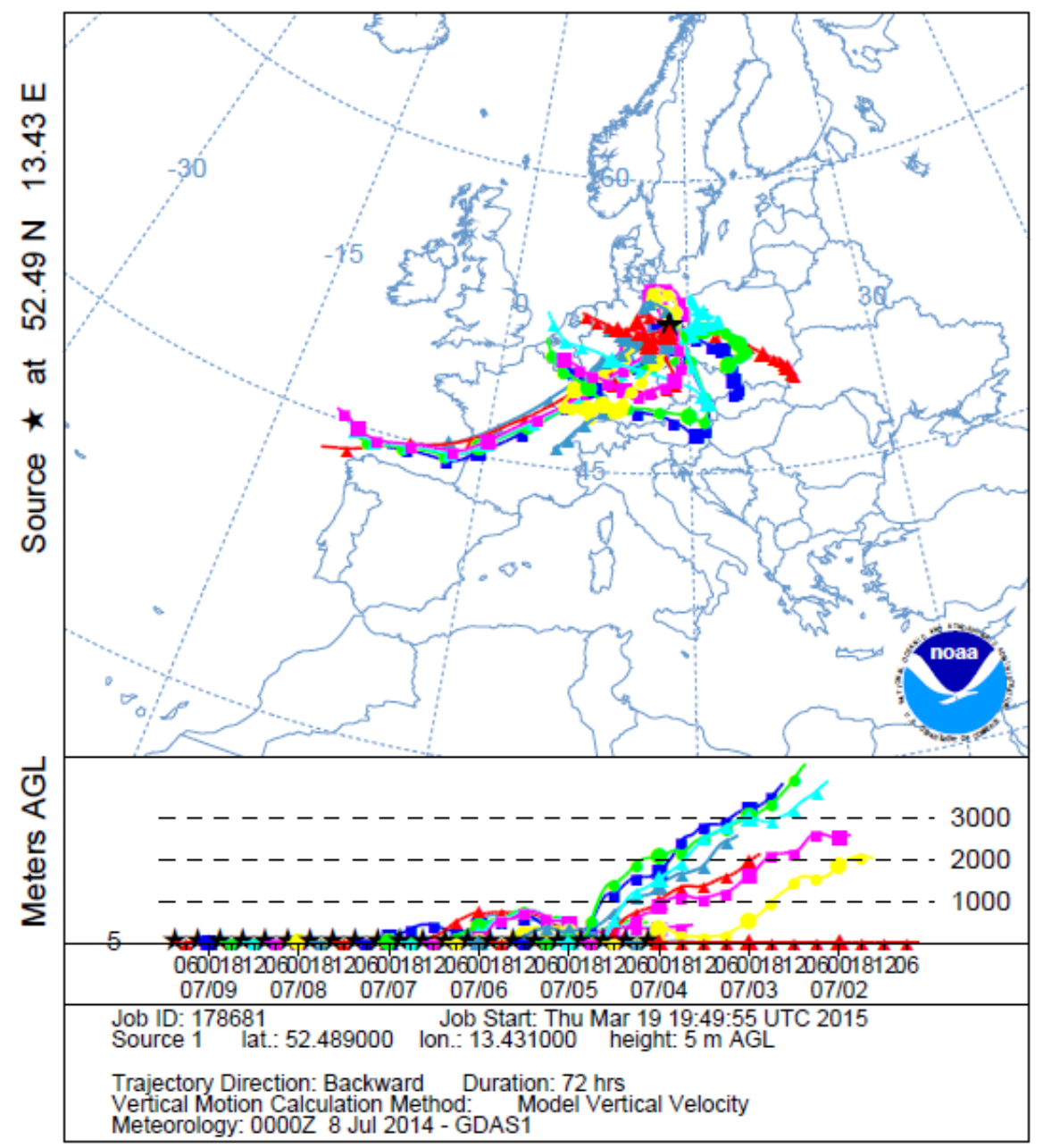


NOAA HYSPLIT MODEL

Backward trajectories ending at 0900 UTC $17 \mathrm{Jul} 14$

GDAS Meteorological Data

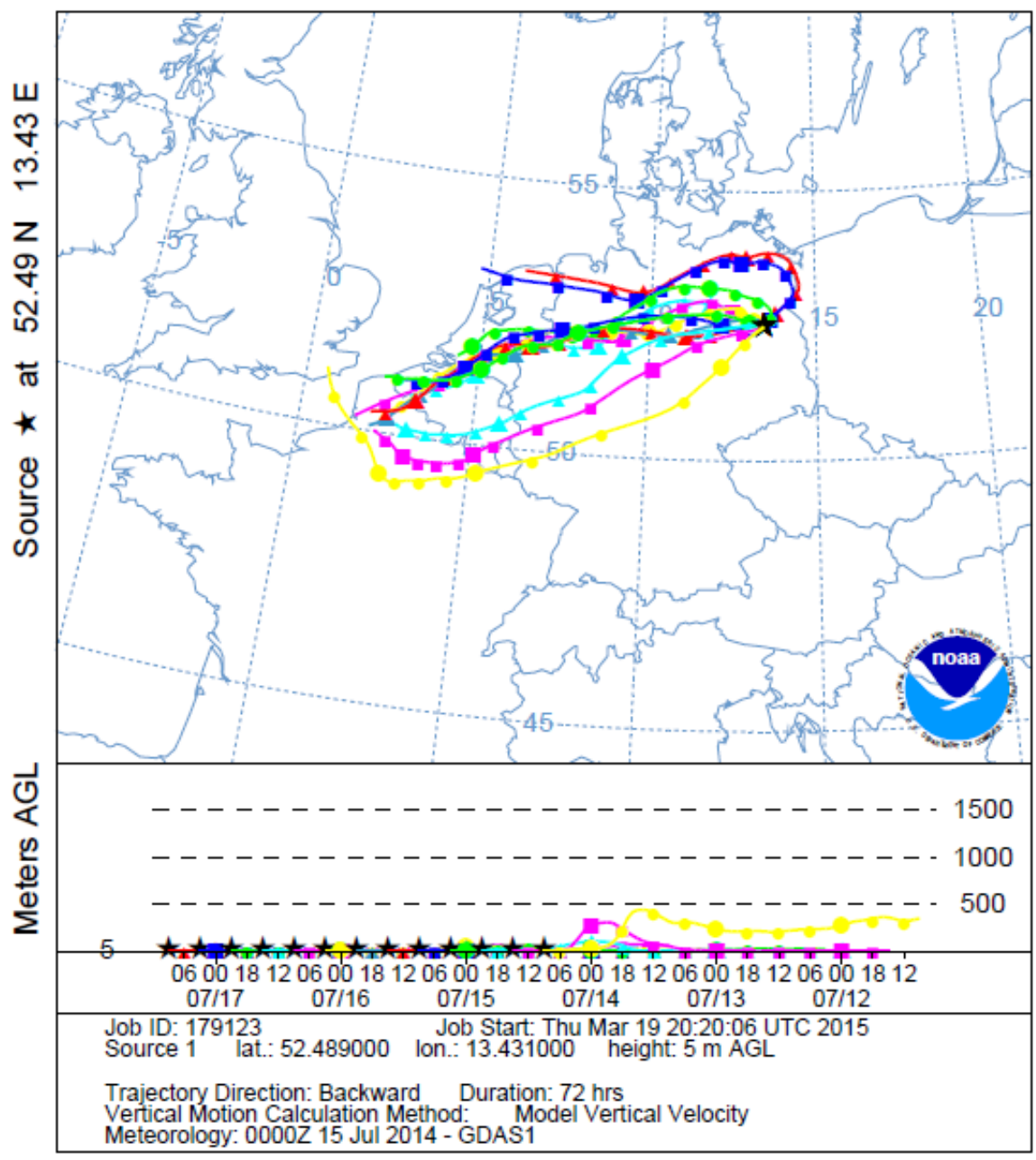


NOAA HYSPLIT MODEL

Backward trajectories ending at 0700 UTC 08 Aug 14

GDAS Meteorological Data

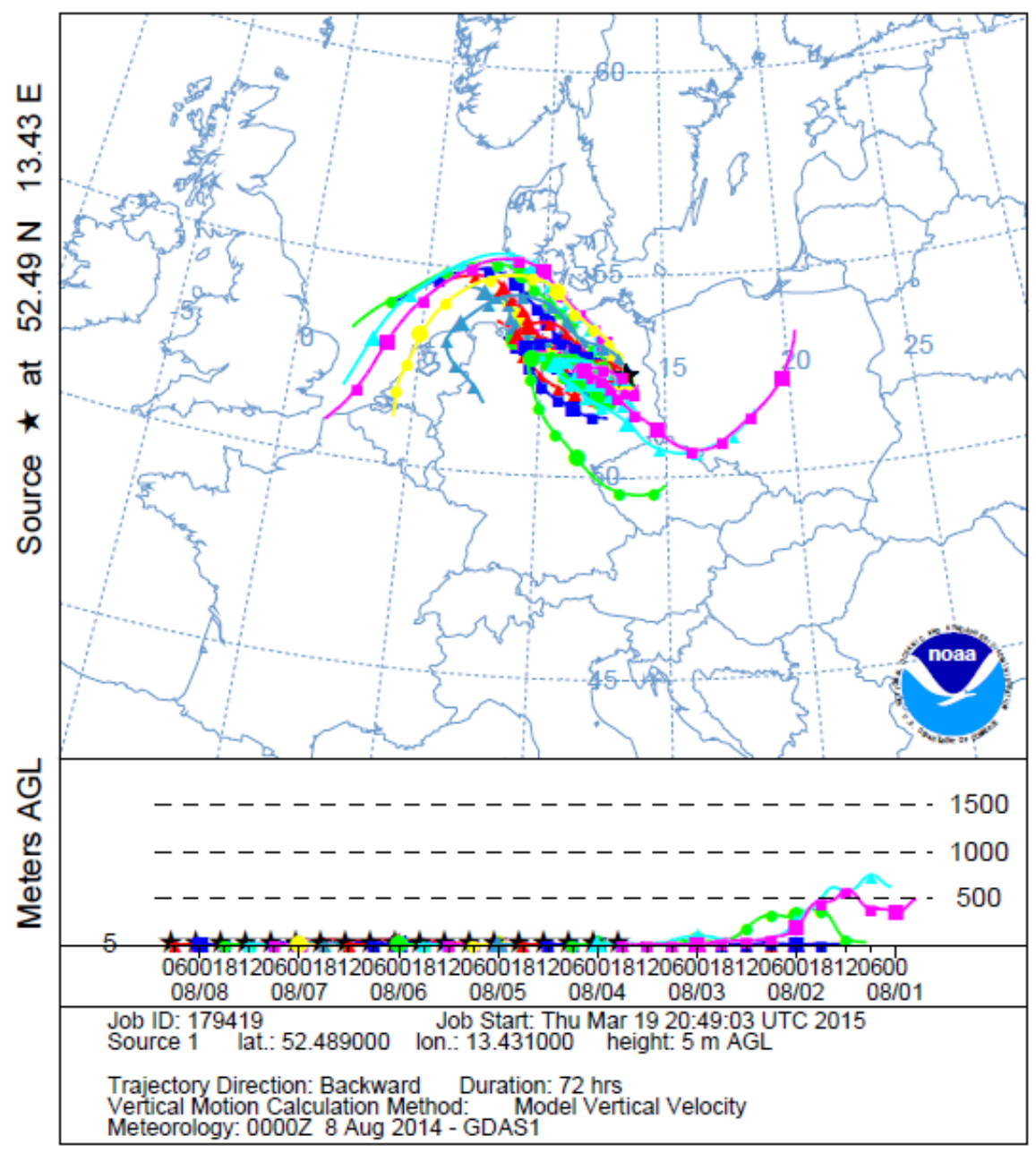


NOAA HYSPLIT MODEL

Backward trajectories ending at 1500 UTC 10 Aug 14

GDAS Meteorological Data

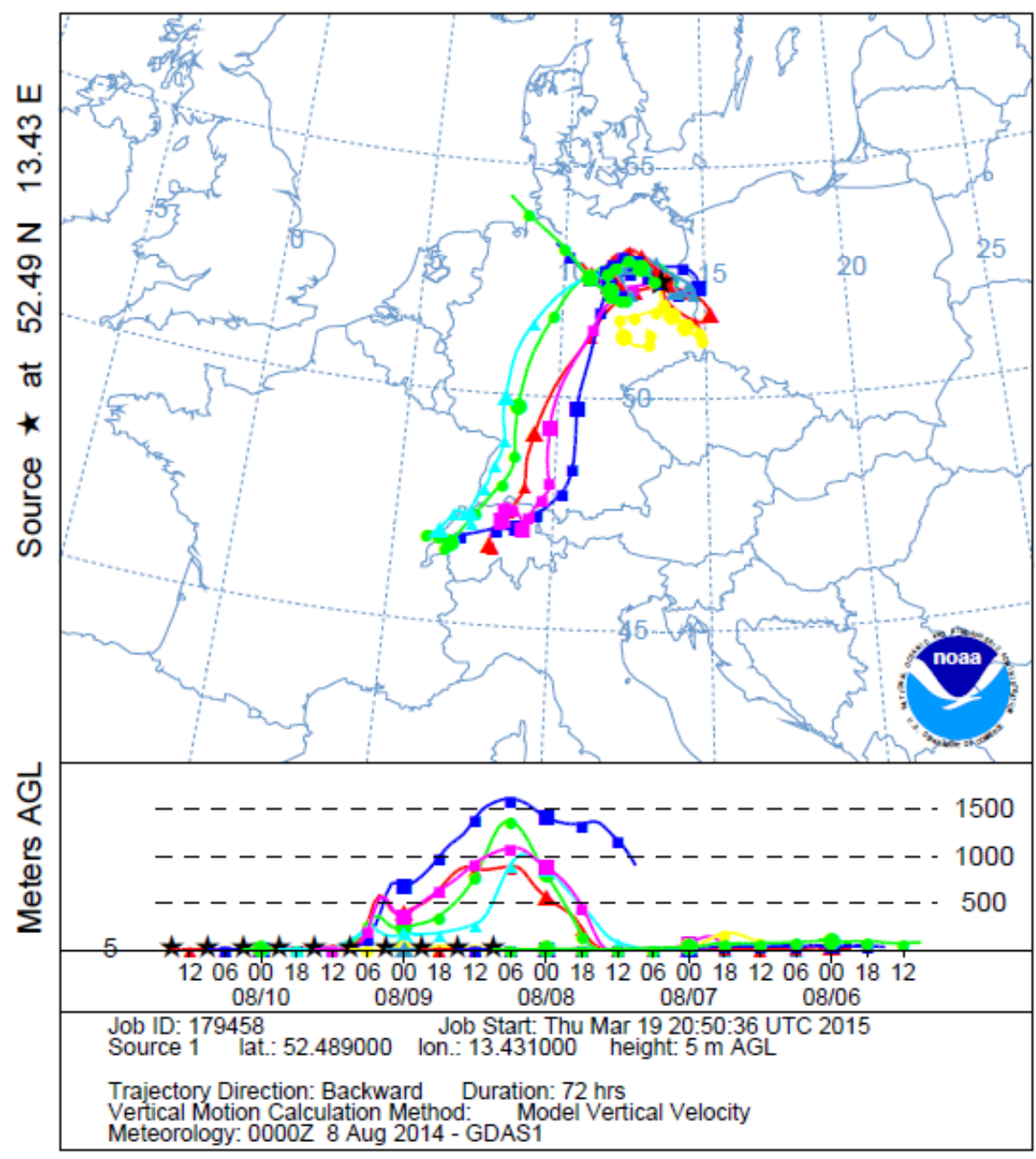


(b) Group B

NOAA HYSPLIT MODEL

Backward trajectories ending at 0800 UTC 14 Aug 14 GDAS Meteorological Data

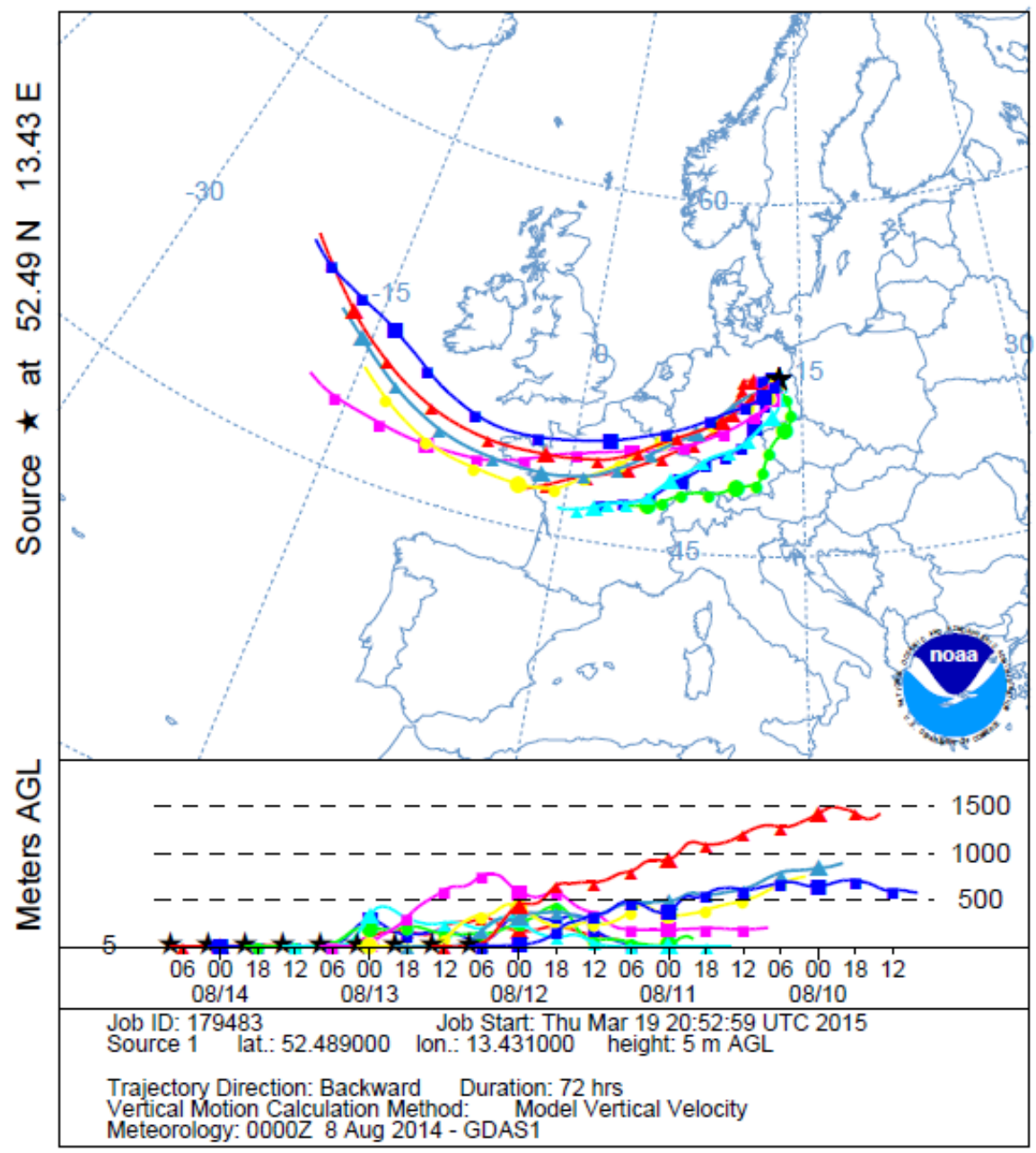


NOAA HYSPLIT MODEL

Backward trajectories ending at 1500 UTC 18 Aug 14

GDAS Meteorological Data

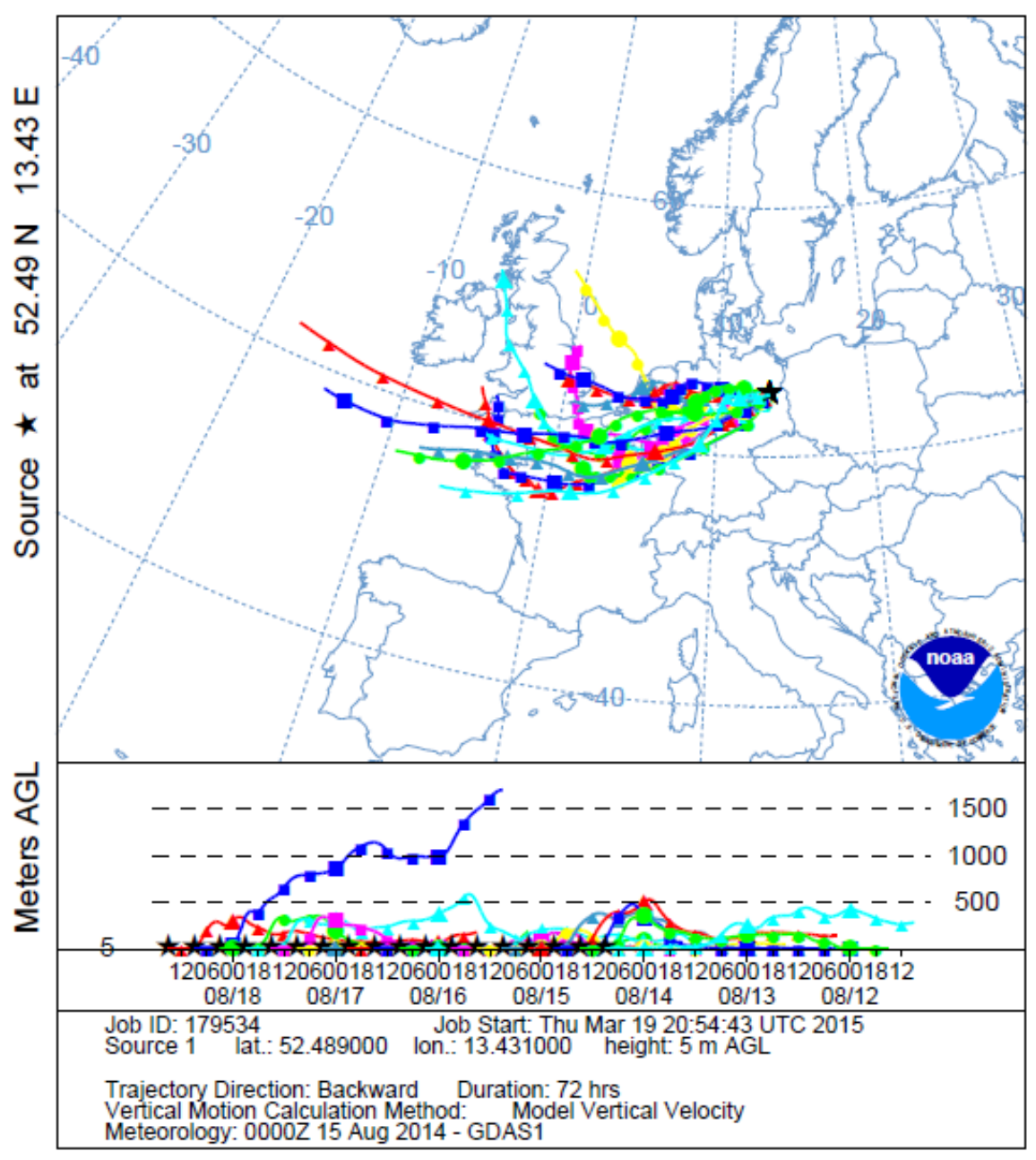


(c) Group C

NOAA HYSPLIT MODEL

Backward trajectories ending at 1000 UTC 20 Jun 14

GDAS Meteorological Data

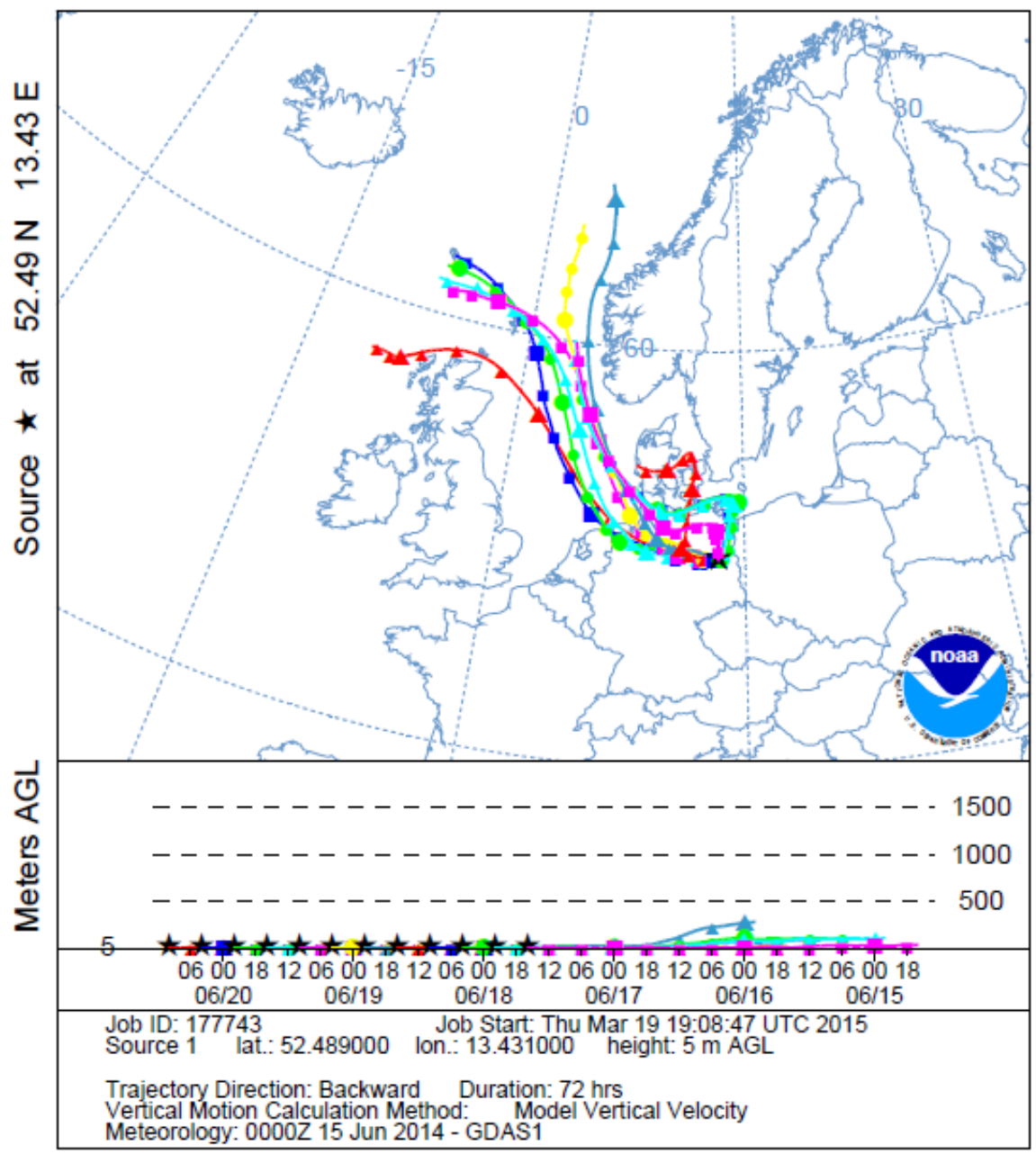


NOAA HYSPLIT MODEL

Backward trajectories ending at 0600 UTC 23 Jun 14

GDAS Meteorological Data

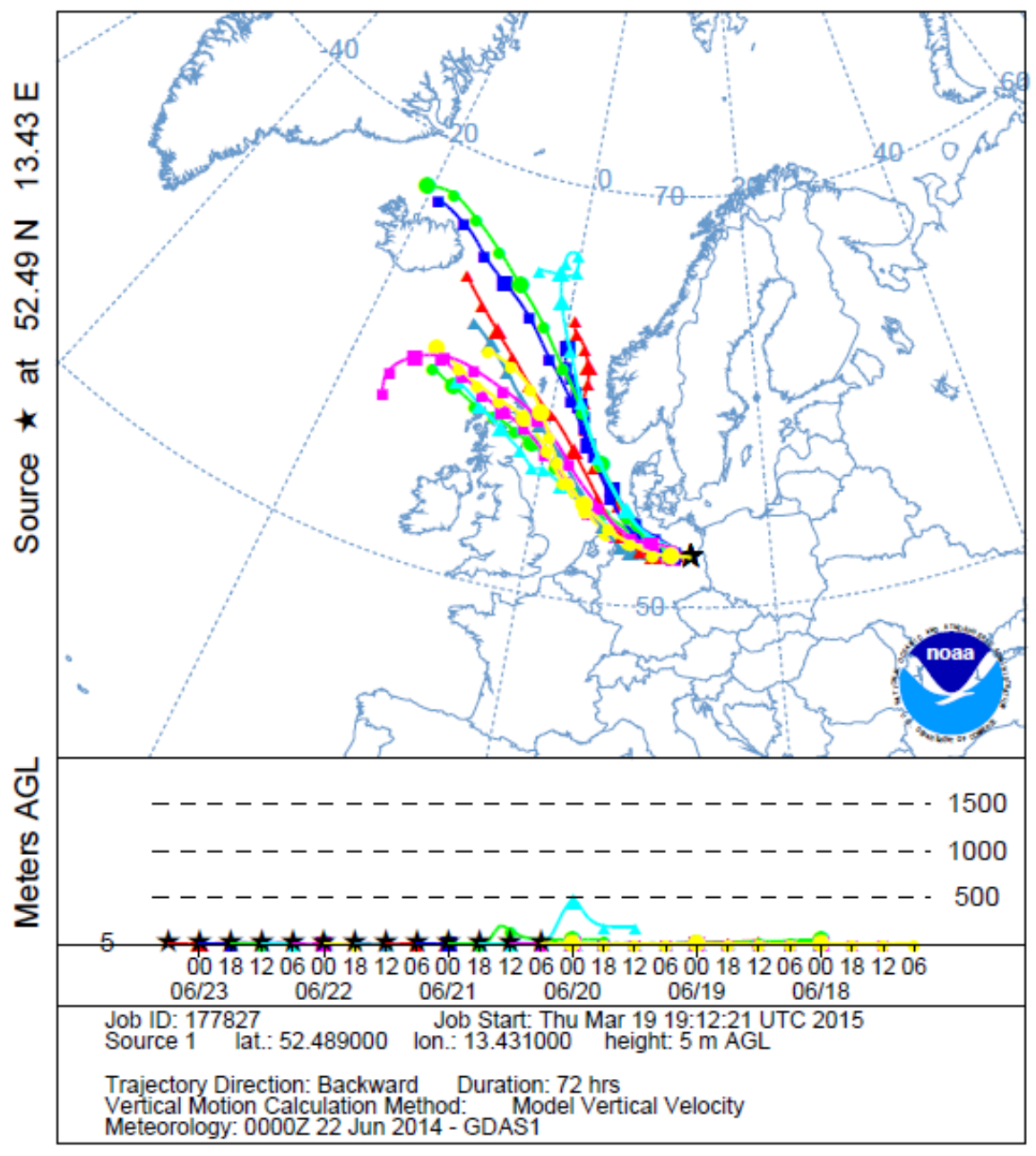


NOAA HYSPLIT MODEL

Backward trajectories ending at 1300 UTC 25 Jun 14

GDAS Meteorological Data

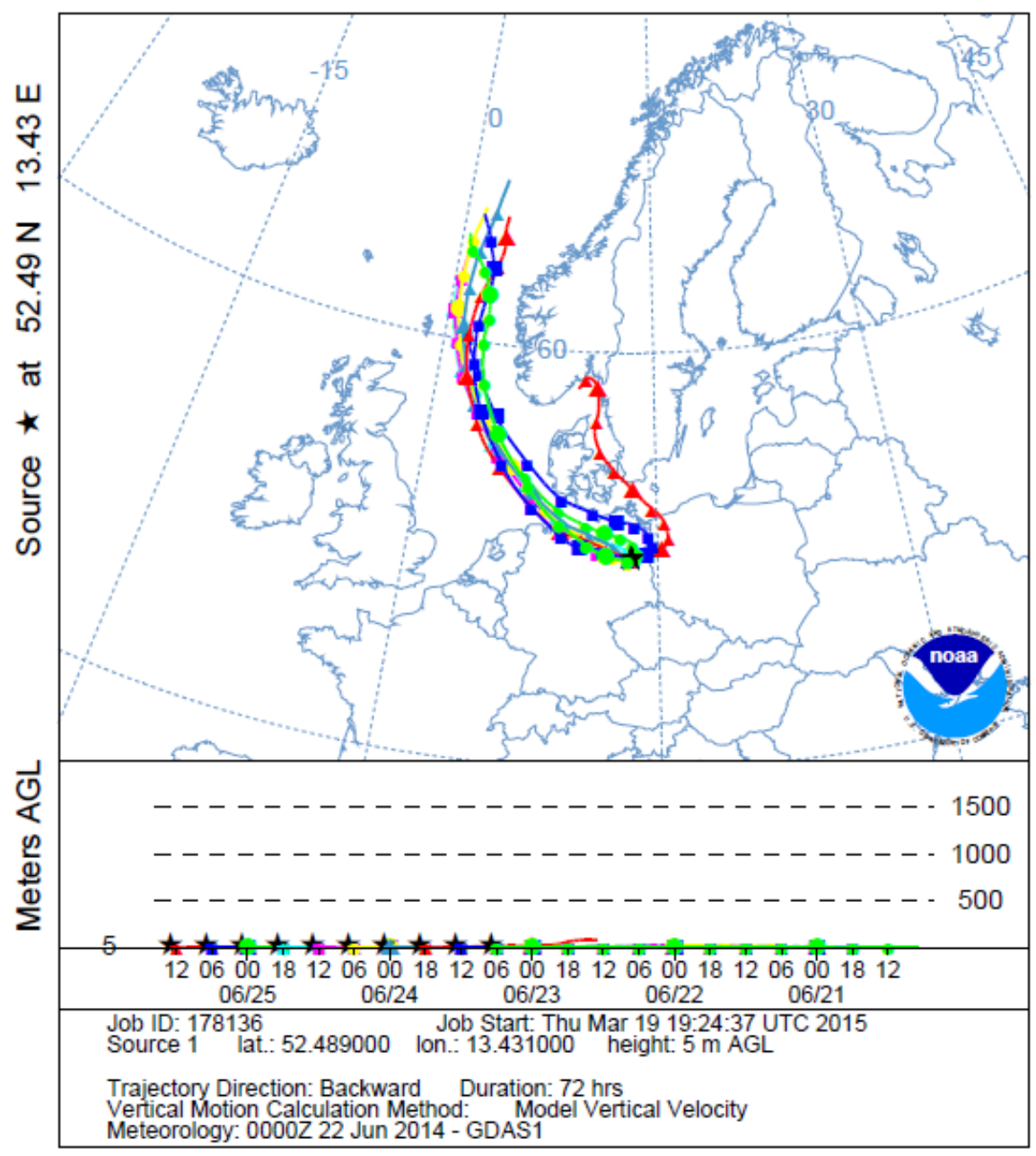


(d) Group D

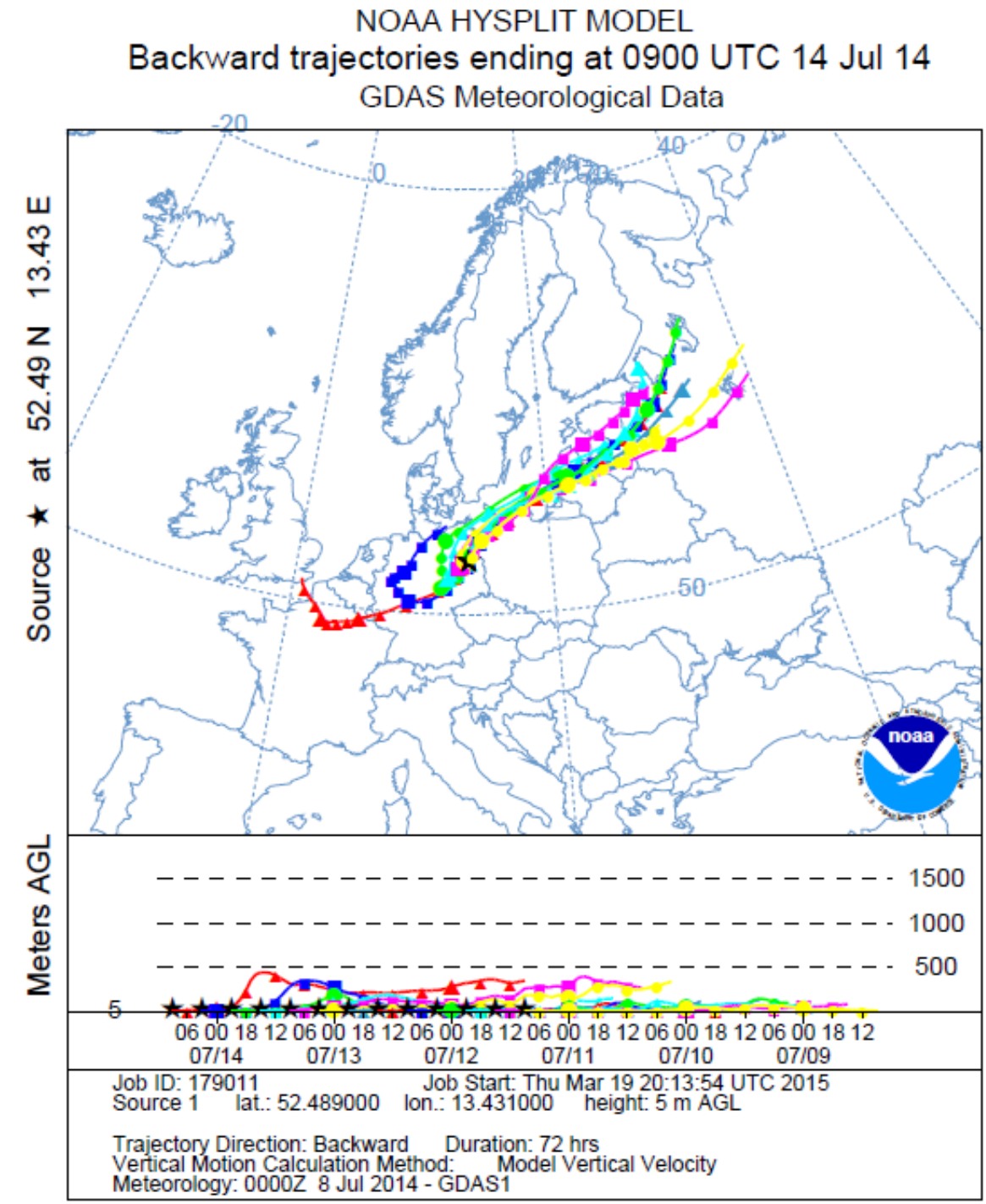


NOAA HYSPLIT MODEL

Backward trajectories ending at 1900 UTC 23 Jul 14

GDAS Meteorological Data

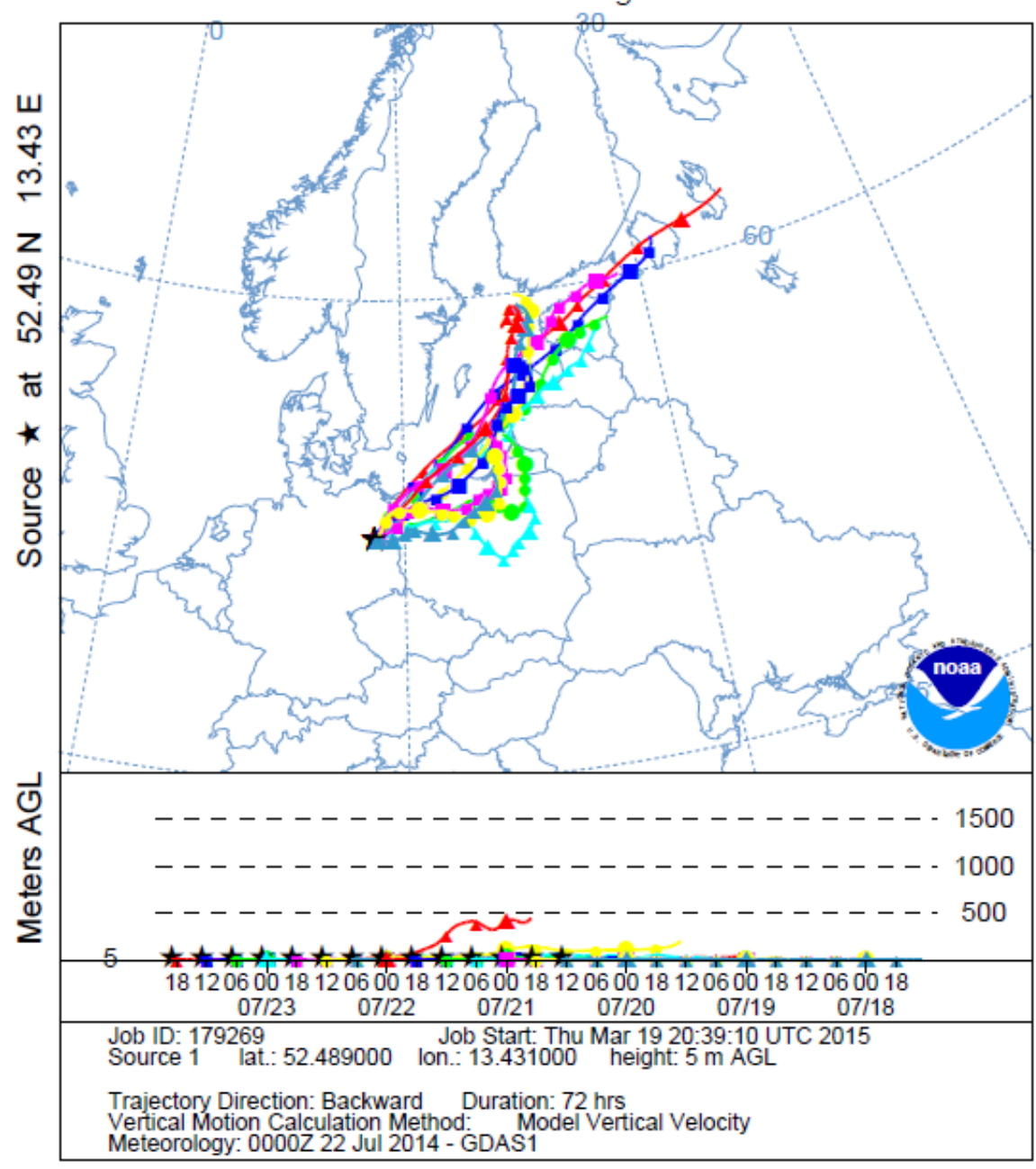


NOAA HYSPLIT MODEL

Backward trajectories ending at 0900 UTC 26 Jul 14

GDAS Meteorological Data

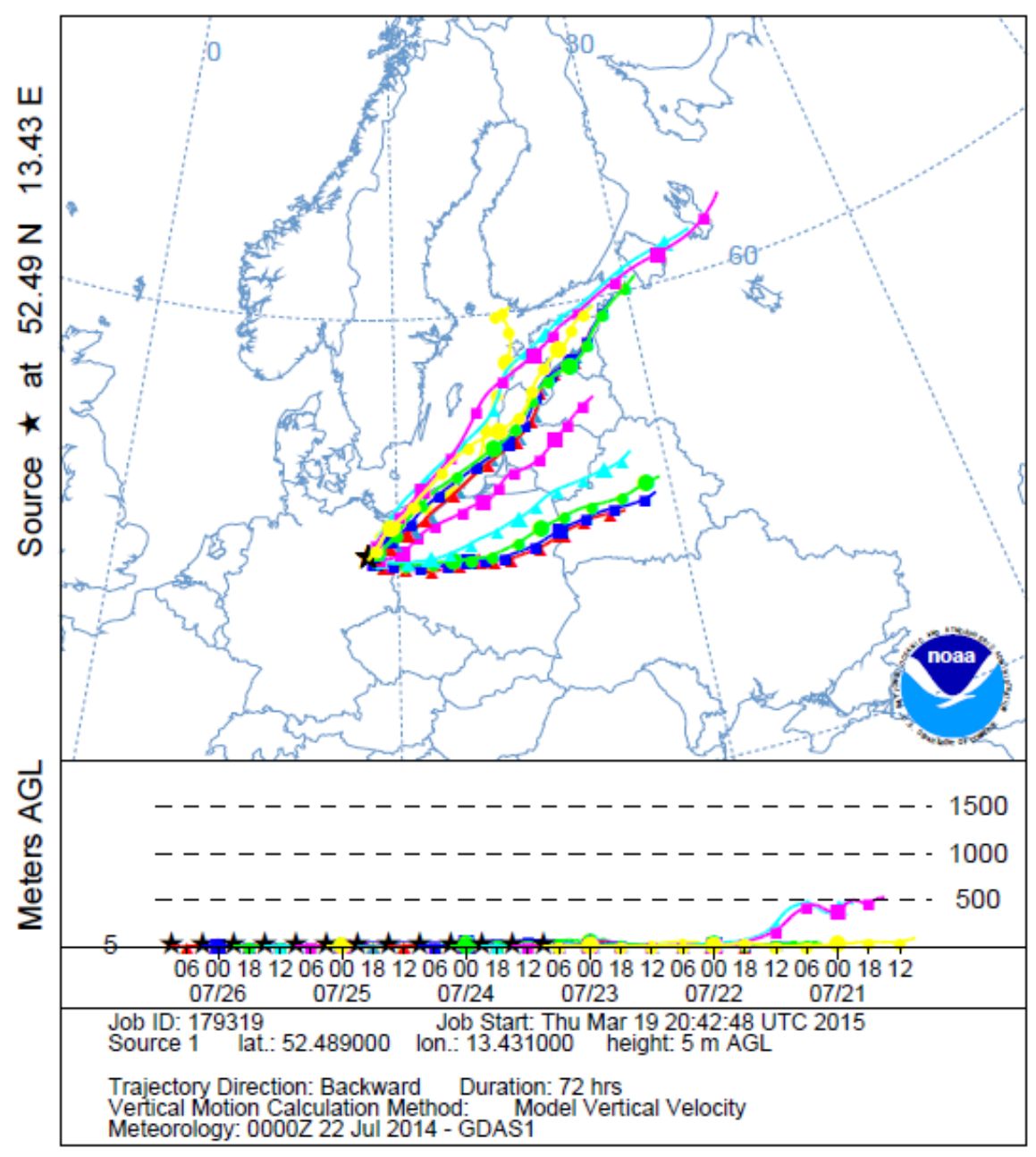


(e) Group E

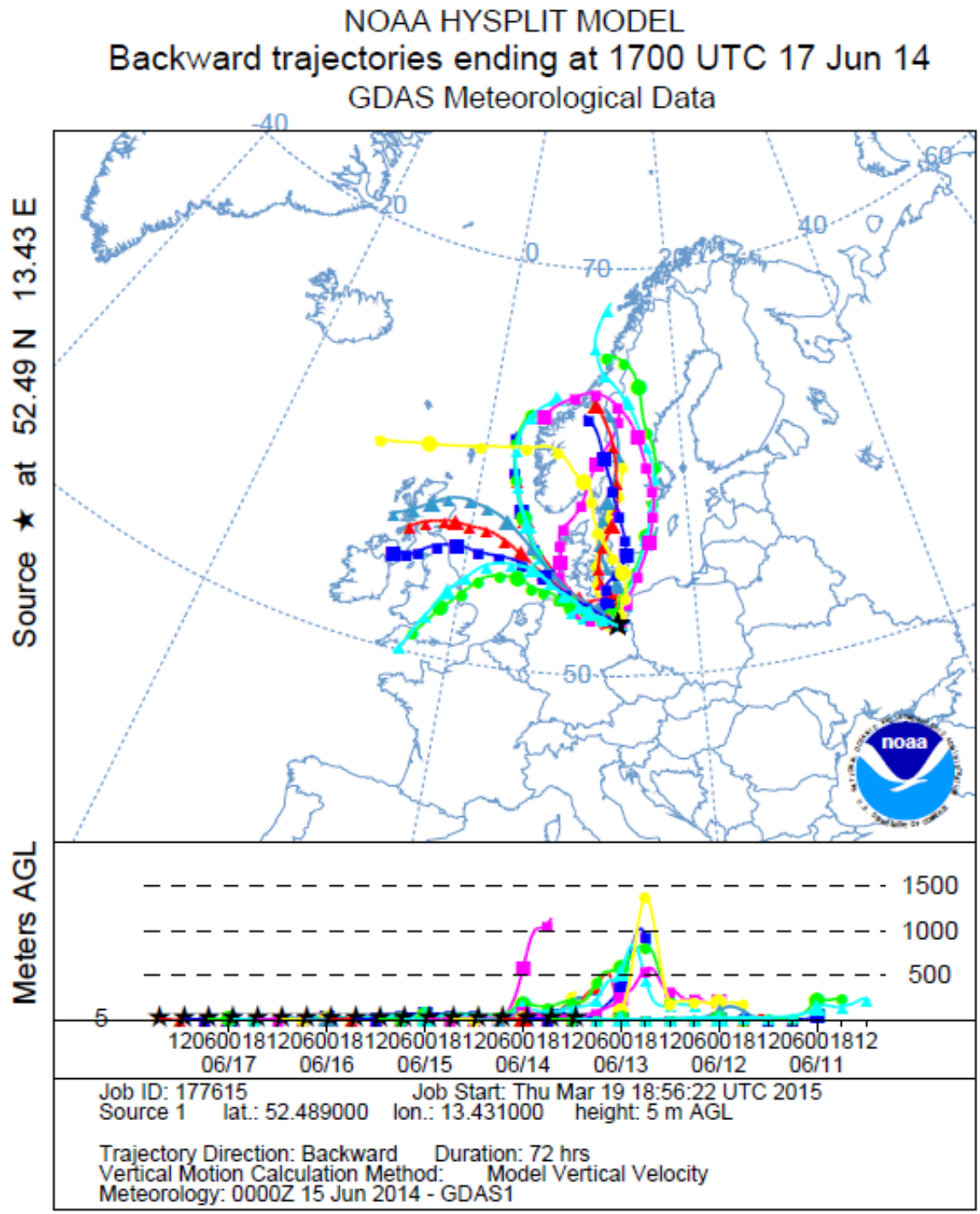




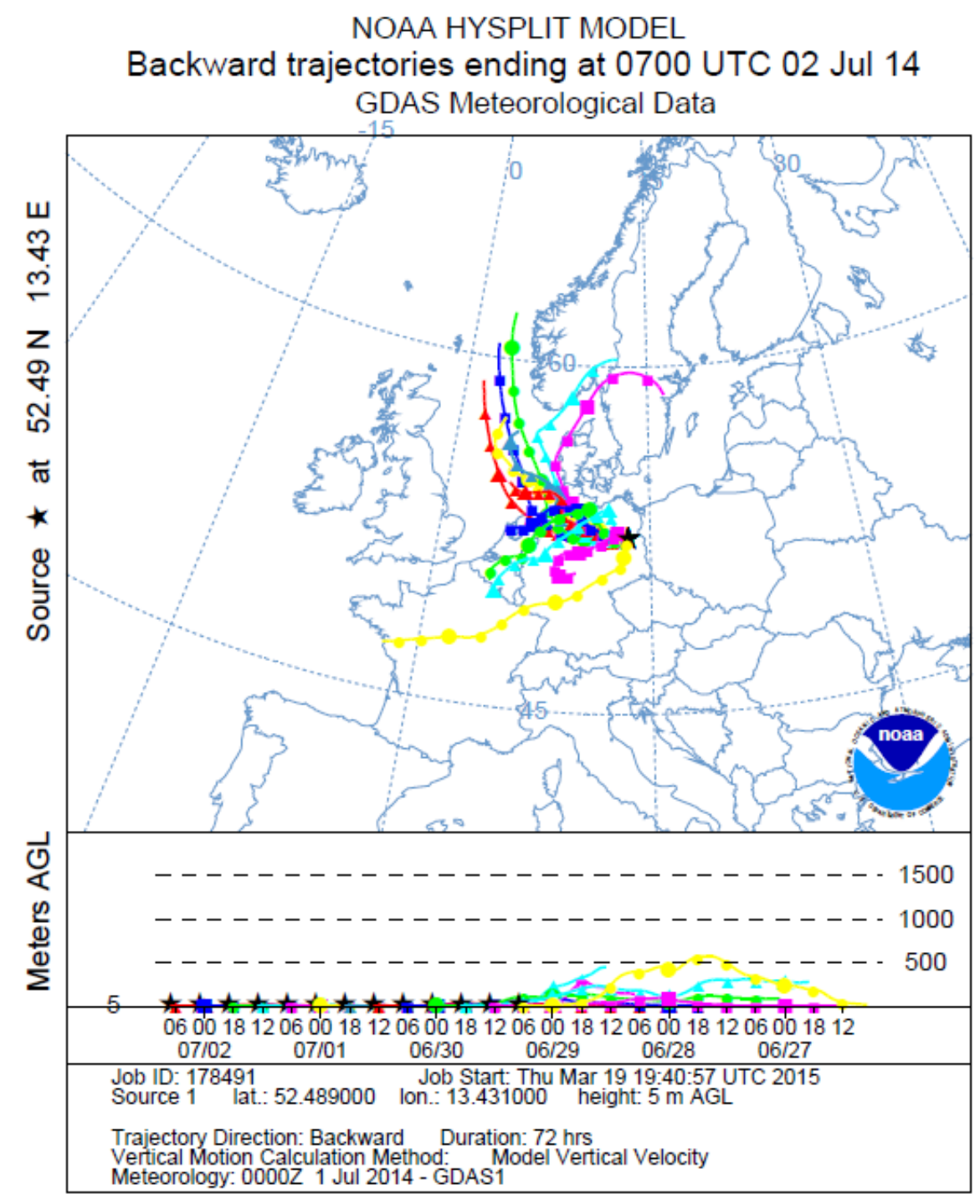


(f) Single filter B17

NOAA HYSPLIT MODEL

Backward trajectories ending at 1600 UTC 29 Jul 14

GDAS Meteorological Data

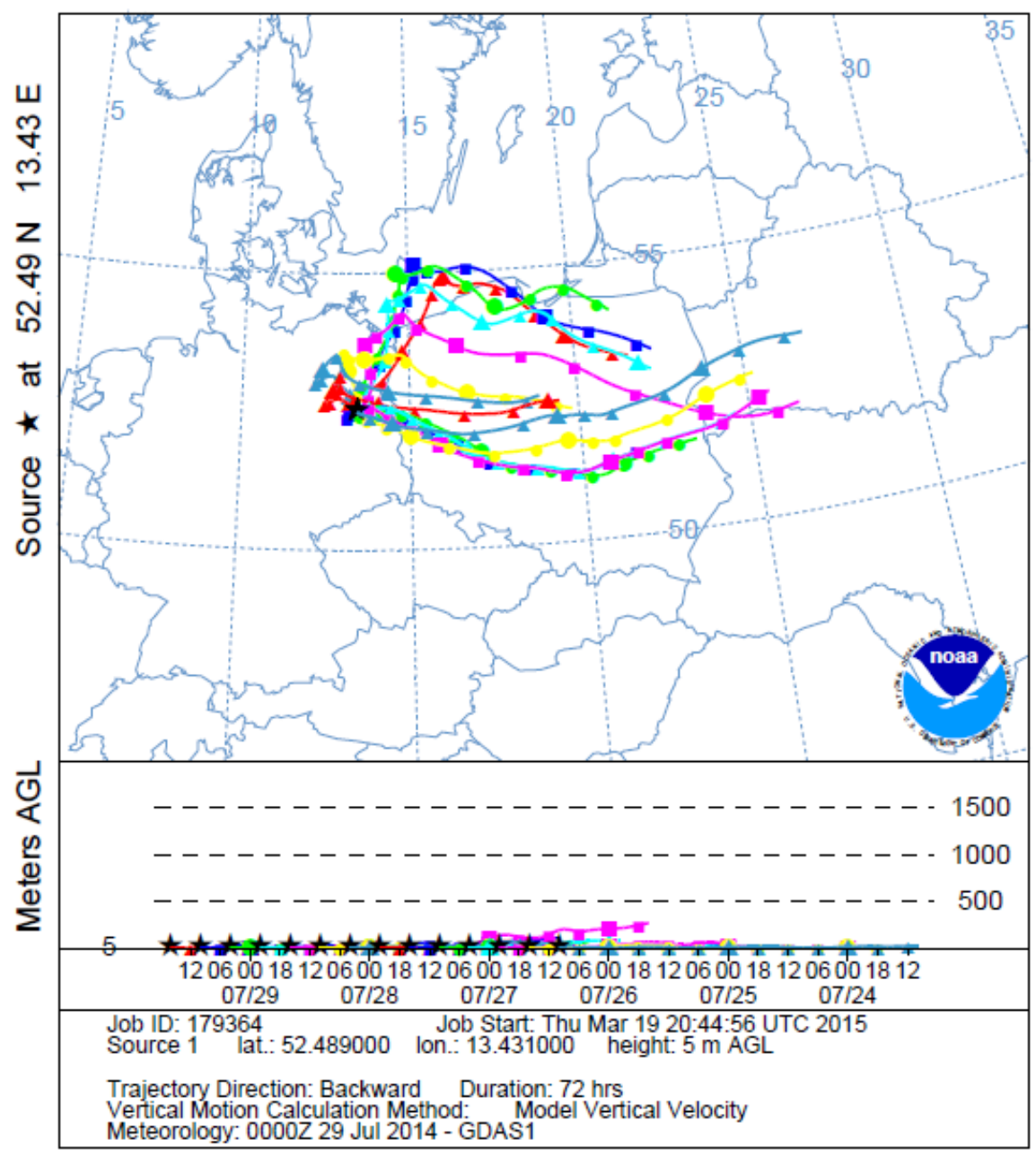


(g) Single filter B19

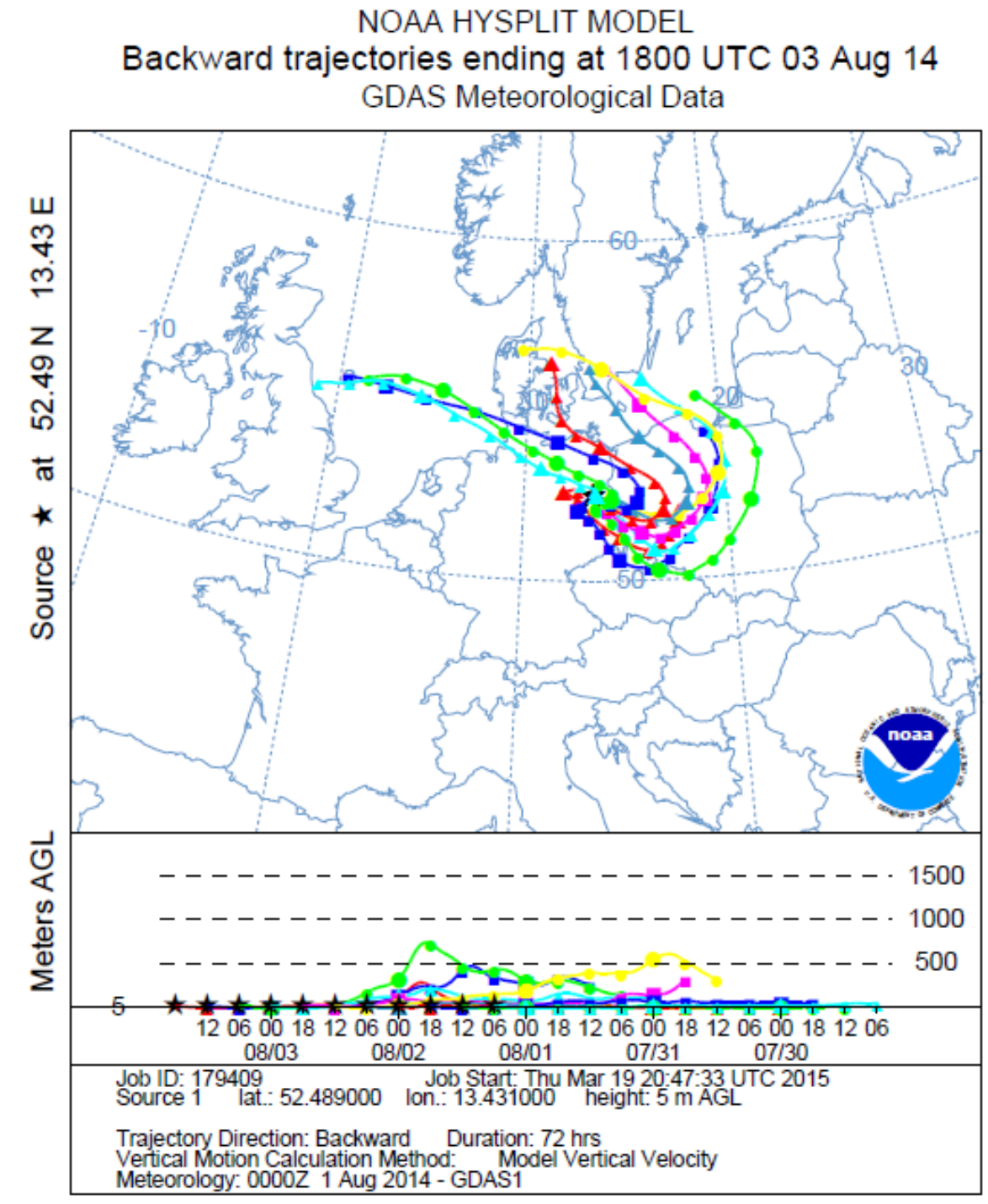


(h) Single filter B30

NOAA HYSPLIT MODEL

Backward trajectories ending at 0700 UTC 01 Sep 14 GDAS Meteorological Data

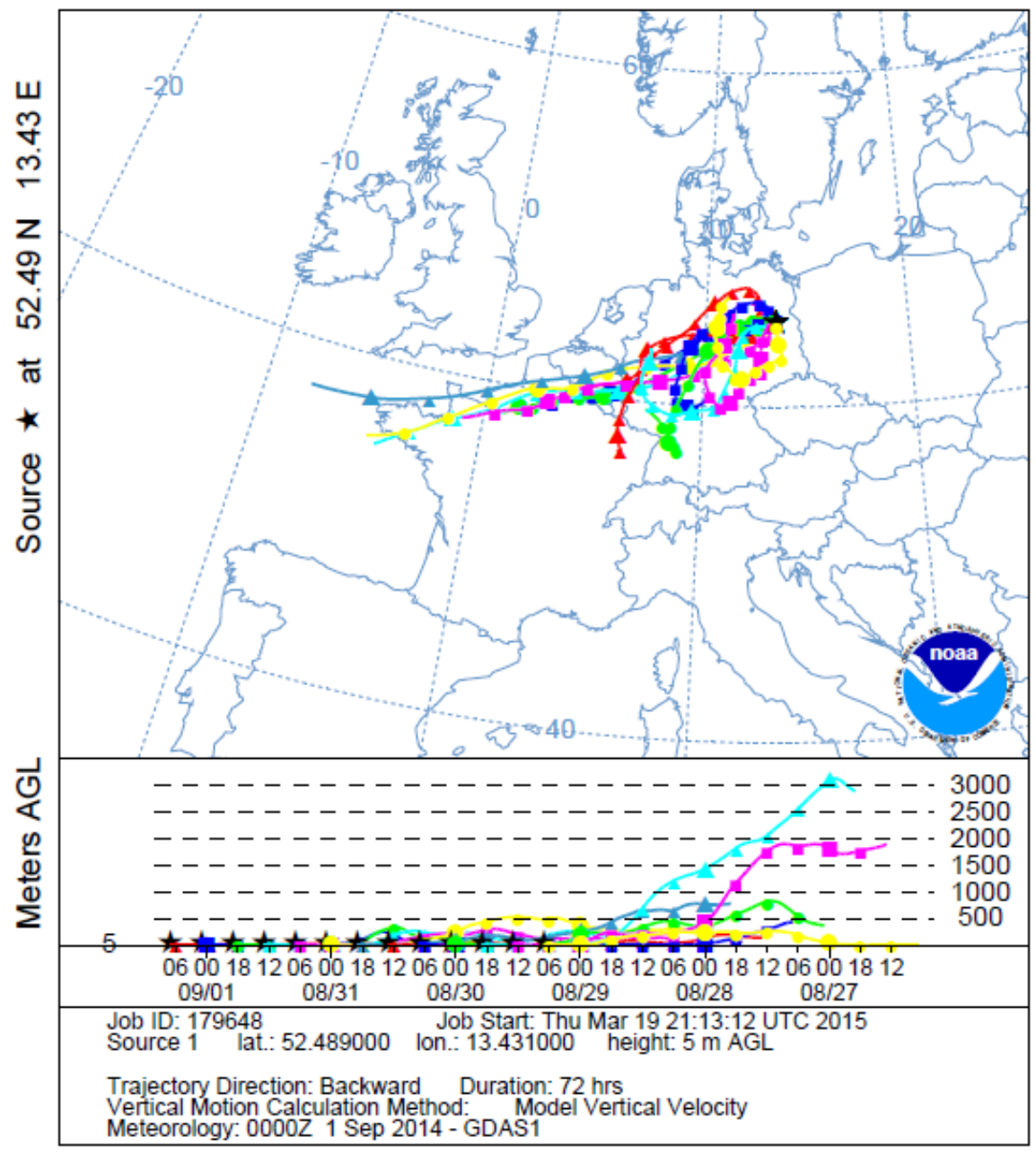

